\title{
Cryo-EM structure of human ATP-citrate lyase in complex with a potent inhibitor
}

Jia Wei, ${ }^{1}$ Silvana Leit, ${ }^{2}$ Jun Kuai, ${ }^{2}$ Eric Therrien, ${ }^{3}$ Salma Rafi, ${ }^{3}$ H. James Harwood Jr, ${ }^{2}$ Byron DeLaBarre, ${ }^{2}$ Liang Tong ${ }^{*}$

${ }^{1}$ Department of Biological Sciences, Columbia University, New York, NY 10027, USA

${ }^{2}$ Nimbus Therapeutics, 130 Prospect Street, Suite 301, Cambridge, MA 02139, USA

${ }^{3}$ Schrödinger, LLC, 120 West $45^{\text {th }}$ Street, $17^{\text {th }}$ Floor, New York, NY 10036, USA

e-mail addresses: Jia Wei, j.wei@columbia.edu Liang Tong, ltong@columbia.edu

ATP-citrate lyase (ACLY) is a central metabolic enzyme and catalyzes the ATP-dependent conversion of citrate and CoA to oxaloacetate and acetyl-CoA. The acetyl-CoA product is crucial for fatty acid metabolism, cholesterol biosynthesis, and protein acetylation and prenylation. There is significant recent interest in ACLY as a target for anti-cancer drugs, since many cancer cells depend on its activity for proliferation. ACLY is also a target against dyslipidemia and hepatic steatosis, with a compound currently in phase 3 clinical trials. Many ACLY inhibitors have been reported over the years, but most of them have weak activity. We report here the development of a series of low nanomolar, small molecule inhibitors of human ACLY. Importantly, we have determined the structure of full-length human ACLY homo-tetramer in complex with one of these inhibitors (NDI-091143) by cryo-electron microscopy, revealing an unexpected mechanism of inhibition (Fig. 1). The compound is located in an allosteric, mostly hydrophobic cavity next to the citrate binding site, and requires extensive conformational changes in the enzyme that indirectly disrupt citrate binding. The observed binding mode is supported by and explains the structureactivity relationships on these compounds. This allosteric site greatly enhances the 'druggability' of ACLY and represents an attractive target for developing new ACLY inhibitors.
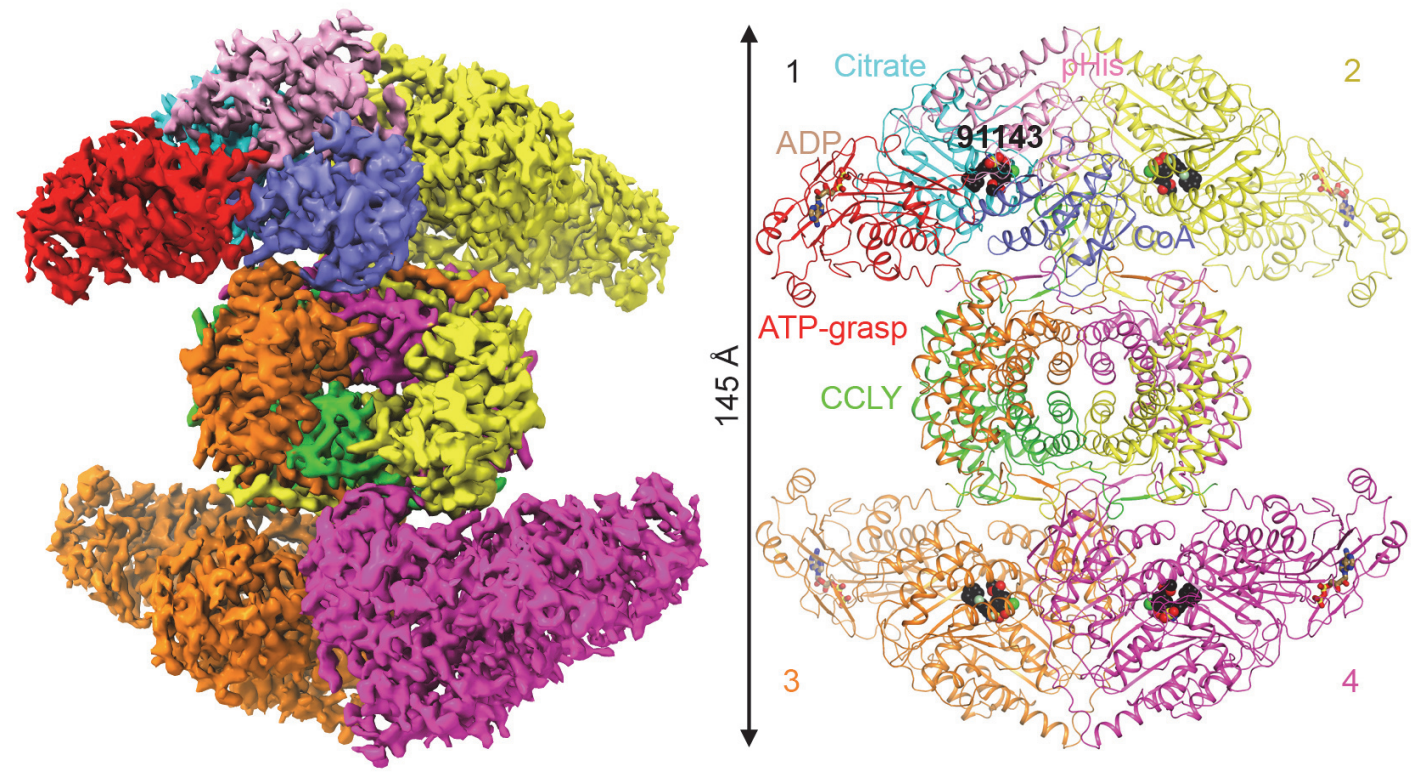

Fig. 1. Cryo-EM map and atomic model of human ATP-citrate lyase tetramer in complex with NDI091143 and ADP.

Reference:

[1] Wei, J. et al. (2019) Nature, doi: 10.1038/s41586-019-1094-6. [Epub ahead of print] 